\section{A Mass Outbreak of Group A Streptococcal Pharyngitis among Policemen in Fukuoka}

Generally speaking, little is known about the so-called foodborn epidemic of group A streptococcal pharyngitis in Japan, and only a few reports are available $(1,2)$. Reportedly, the introduction of mechanical refrigeration and milk pasteurization largely put an end to foodborn outbreaks of streptococcal infection in western countries, but occasional foodborn outbreaks of streptococcal pharyngitis has been observed still in recent years (3).

Fukuoka prefectural policemen were asked to assist for a safe and smooth operation of the 30th Annual Meeting of Asian Development Bank held in Fukuoka from May 11-13th, 1997. They were very busy during this period and were supplied lunch by two food catering companies (companies $\mathrm{N}$ and $\mathrm{S}$ ). Although the meeting was successful and fruitful, unfortunately it was followed by a sudden onset of symptoms such as sore throat, fever, general malaise, joint and muscle pain among policemen engaged in this operation (36.2\%).

The questionnaire gathered from all policemen engaged demonstrated that these symptoms occurred mainly between May 12-14, which suggested that the development of symptoms was induced by a single factor exposure. The occurrence ratio of symptoms was apparently higher in policemen who had taken lunch supplied by company $\mathrm{N}$ (623/877 vs 99/608).

Typical objective findings found in patients who attended the Fukuoka Prefectural Police Clinic were enlargement of tonsils with white coating on them, leukocytosis, positive C-reactive protein (CRP) and low serum iron levels. Serological tests (ASK, ASO) performed on paired serum were positive in 77/196 (39.3\%). Throat culture performed in three different clinics revealed Group A, $\beta$-streptococcus; also the omelet which was part of the lunch entree supplied on May 10 by company $\mathrm{N}$ was contaminated by the same bacterium (S. pyogenes A-Group, T type B3264, Health Environmental Institute, Fukuoka). Further, DNA analysis performed on S. pyogenes obtained from both materials showed complete identity in its pattern (National Infectious Disease Institute).

These findings led us to the conclusion that a mass outbreak of group A streptococcal pharyngitis had occurred due to the intake of omelet which was one of lunch ingredients contaminated with $S$. pyogenes. It is regretful that the mechanism of contamination with this bacterium remains unclear. This foodborn outbreak of group A streptococcal pharyngitis in Fukuoka emphasizes that careful attention should be paid to this kind of outbreak from the medical and hygienic standpoint.

\section{Takashi Yамамото, Tatsurou IYAnaga* and Tsunenori MIYAKE**}

From *the Fukuoka Prefectural Police, Department of Welfare, Health Care Unit and Clinic and **Miyake Medical Hospital, Fukuoka

Reprint requests should be addressed to Dr. Takashi Yamamoto, the Fukuoka Prefectural Police, Department of Welfare, Health Care Unit and Clinic, 7-7, Higashikoen, Hakata-ku, Fukuoka 812

\section{References}

1) Okuyama Y. Epidemiological study of a food-borne epidemic of pharyngitis due to Group A, T type 12 streptococcus. Kansenshogaku Zasshi 56: 1173, 1982.

2) Kashiwagi Y, Hori M, Bushimata K, et al. A foodborne outbreak of streptococcal sore throat occurred in Tokyo, 1983. Kansenshogaku Zasshi 60: $673,1986$.

3) Shemesh E, Fishel T, Goldstein N, Alkan M, Livneh A. An outbreak of foodborne streptococcal throat infection. Isr J Med Sci 30: 275, 1994 (see comments)

\section{Nasogastric Tube Feeding and Esophageal Disorders}

Norton and colleagues reported that percutaneous endoscopic gastrostomy feeding is superior to nasogastric tube feeding after acute dysphagic stroke in a prospective comparison of mortality and nutritional state (1). We recommend gastrostomy feeding for another reason. Between January 1995 and June 1995, detailed esophagoscopy (Olympus GIF XQ) was prospectively performed on 23 dysphagic patients (mean age: 76.7 , male: 3 , female: 20 ) who were fed by 16 Fr nasogastric tubes for an average of 10.6 months (one day to 30 months). Various esophageal disorders were recognized in 11 patients (48\%). Of these disorders various patterns were found, such as a form of reflux esophagitis, multiple erosions, oval or longitudinal ulcers distributed from the upper to lower esophagus and a cardiac tear injury (Table 1). The duration of intubation was from one day to 30 months (average 11.8 months). It appeared to be unrelated to the occurrence of esophageal disorders. In such patients without reflux esophagitis, these disorders healed within one week after gastrostomy feeding. Although most patients with esophageal disorder also had anemia, they recovered gradually after gastrostomy feeding. Nasogastric tube feeding, although easy, is not recommended because it causes various esophageal disorders and anemia.

Table 1. Esophageal Disorders Caused by Nasogastric Intubation

\begin{tabular}{clcc} 
Patients & Esophageal lesion & Esophageal location & Duration \\
\hline 1. $79 \mathrm{~F}$ & RE $(\mathrm{A})^{\mathrm{a}}$ & lower & 30 months \\
2. $84 \mathrm{~F}$ & $\mathrm{RE}(\mathrm{A})$ & lower & 24 months \\
3. $80 \mathrm{~F}$ & $\mathrm{RE}(\mathrm{B})^{\mathrm{b}}$ & lower & 24 months \\
4. $80 \mathrm{~F}$ & multiple erosions & lower & 1 day \\
5. $83 \mathrm{~F}$ & multiple erosions & middle & 2 weeks \\
6. $78 \mathrm{~F}$ & multiple erosions & middle & 5 months \\
7. $79 \mathrm{~F}$ & oval ulcers & upper & 2 weeks \\
8. $90 \mathrm{~F}$ & oval ulcers & middle & 18 months \\
9. $68 \mathrm{M}$ & longitudinal ulcers & lower & 1 month \\
10. $59 \mathrm{M}$ & longitudinal ulcers & lower & 21 months \\
11. $78 \mathrm{~F}$ & tear injury & cardia & 1 month
\end{tabular}

a: Reflux esophagitis grade A with Los Angeles classification. b: Reflux esophagitis grade B with Los Angeles classification.

\section{Hirofumi Miyoshi, Mitsuhiro Yata*, Noriaki Matsuo*, Yoshiko Sameshima* and Toshimitsu Harima**}

The Internal Medicine, Kobe Teishin Hospital, Chuo-ku, Kobe 651, *The Internal Medicine and **the Department of Radiology, Seifuen Imai Hospital, Inagawa, Hyogo 666-02

\section{Reference}

1) Norton B, Homer-Ward M, Donnelly MT, Long RG, Holmes GKT. A randomised prospective comparison of percutaneous endoscopic gastrostomy and nasogastric tube feeding after acute dysphagic stroke. BMJ 312: 13, 1996. 